University of Nebraska - Lincoln

DigitalCommons@University of Nebraska - Lincoln

December 1999

\title{
Langmuir monolayers as disordered solids: Disorder and elastic fluctuations in mesophases
}

Tadeusz Luty

Institute of Physical and Theoretical Chemistry, Technical University of Wrocław, Poland

Craig J. Eckhardt

University of Nebraska - Lincoln, ceckhardt1@unl.edu

Jacques Lefebvre

Laboratoire de Dynamique et Structures des Materiaux Moleculaires, Universite de Lille 1, France

Follow this and additional works at: https://digitalcommons.unl.edu/chemistryeckhardt

Part of the Chemistry Commons

Luty, Tadeusz; Eckhardt, Craig J.; and Lefebvre, Jacques, "Langmuir monolayers as disordered solids: Disorder and elastic fluctuations in mesophases " (1999). Craig J. Eckhardt Publications. 6.

https://digitalcommons.unl.edu/chemistryeckhardt/6

This Article is brought to you for free and open access by the Published Research - Department of Chemistry at DigitalCommons@University of Nebraska - Lincoln. It has been accepted for inclusion in Craig J. Eckhardt Publications by an authorized administrator of DigitalCommons@University of Nebraska - Lincoln. 


\title{
Langmuir monolayers as disordered solids: Disorder and elastic fluctuations in mesophases
}

\author{
Tadeusz Luty \\ Institute of Physical and Theoretical Chemistry, Technical University of Wroctaw, Poland \\ and Laboratoire de Dynamique et Structures des Materiaux Moleculaires, UPRESA no. 8024, \\ U.F.R. de Physique, Universite de Lille 1, France
}

Craig J. Eckhardt

Department of Chemistry and Center for Materials Research and Analysis, University of Nebraska-Lincoln, Lincoln, Nebraska 68588-0304

Jacques Lefebvre

Laboratoire de Dynamique et Structures des Materiaux Moleculaires, UPRESA no. 8024, U.F.R. de Physique, Universite de Lille 1, France

(Received 30 July 1999; accepted 11 September 1999)

\begin{abstract}
Ordering in Langmuir monolayer mesophases is examined using an approach based on the elastic theory of crystals. Molecular tails are modeled as "defects" grafted onto a two-dimensional elastic medium and are characterized by elastic dipoles. It is assumed that disorder in the parent, LS, phase is due to competition between local (within a domain) and global (hexagonal arrangement of domains) structure. By treating the LS phase as a mixture of rectangular and/or oblique domains (rectangular and/or oblique defects within the two-dimensional elastic medium), density fluctuations due to elastic interactions between domains are analyzed. The correlation function for the elastic dipoles is calculated and the elastic interactions' renormalization of the elastic properties of mesophases is analyzed. Results are shown to be compatible with very recent experiments on microscopic and macroscopic elasticity of the monolayers as well as those on positional disorder in LS and S phases. Kinetic aspects of the elastic response are considered, as is the contribution of the elastic domains' reorientations to x-ray diffuse scattering. (C) 1999 American Institute of Physics. [S0021-9606(99)70446-0]
\end{abstract}

\section{INTRODUCTION}

Molecular monolayers at the air-water interface exhibit very rich thermodynamic behavior. They form a variety of phases with different degrees of translational and orientational disorder from gaslike to solidlike phases. ${ }^{1}$ The surface pressure-temperature $(\pi-T)$ diagrams of saturated fatty acids allow the establishment of a generic phase diagram. ${ }^{2,3}$ The current view is that the phase behavior of a monolayer displays mesomorphic and solid states and that the subtle and almost continuous changes between these phases even admit amorphous states. The richness of phases indicates that the monolayers are frustrated systems where local and global structures compete as do their respective equilibria. Although there are many factors that may lead to detailed structure in the phase diagram arising from differences in translational and orientational order, predominant are the chemical nature, shape, and flexibility of the amphiphiles. In the simplest picture, a frustration arises because the crosssectional area of the head groups is different, in general, from that of the attached alkane chains, making it impossible to fill space without introducing some strain into the layer configuration, or the molecule itself.

There are ongoing experimental, theoretical, and numerical attempts to gain knowledge about the ordering and molecular nature of the different phases and the transitions between them. Current theoretical approaches, almost exclusively, emphasize similarities of the monolayers to liquid-crystalline rather than crystalline properties. Within this framework, it has been suggested that mesophases of Langmuir monolayers observed in high-temperature regimes are hexatic phases that display long-range orientational (algebraically decaying) and short-range translational (exponentially decaying) order. Crystalline phases that have algebraically decaying translational order are observed at low temperatures.

In recent years there has been impressive progress in experimental structural determinations, although there is still unresolved ambiguity in the assignment of the phases as crystalline or mesophase. ${ }^{4}$ Apart from the fact that the hexatic phase has been proposed for purely 2D systems, hexatic order has not been directly observed for Langmuir monolayers. Moreover, grazing incidence x-ray diffraction experiments have shown the monolayers to be crystalline in both compressed and uncompressed states, ${ }^{5}$ and, very recently, this has also been shown for high-temperature (LS) and low-temperature (S) phases. ${ }^{6}$ In recent elegant studies of the shear elasticity of monolayers, ${ }^{7,8}$ it has been confirmed that these phases are more crystalline in nature, rather than hexatic. These findings strongly support our previously introduced model of Langmuir monolayers as disordered solids, ${ }^{3,9}$ as do other studies ${ }^{10,11}$ that demonstrate mesophases can be treated as two-dimensional (2D) solids. 
The solid state approach that we advocate is based on a view that the intrinsic head-tail asymmetry of Langmuir monolayer molecules makes the system quasi-2D rather than strictly 2D, the latter condition being that for which hexatics are solely defined. Hexatic structure obtains as a result of exponential decay of the displacement-displacement correlation function in 2D systems comprised of objects without rotational (internal) degrees of freedom. Molecular monolayers are quite different. The molecules interact with the aqueous subphase by hydrogen bonding of the head groups and the molecular tails are orientationally flexible. It has been shown by $\mathrm{x}$-ray diffraction studies, that the ordering of head groups on the water surface differs from that of the molecular tails in the air (viz. the geometric frustration mentioned above). ${ }^{12}$ This coincides with our view that Langmuir monolayers can be modeled by a strain field of ordered head groups and molecular tails with orientational freedom (e.g., tilt and backbone orientation). Crystalline phases are characterized by crystallinity of both head groups and tails, while in liquid phases both head groups and molecular tails are fluidized. The melting of a crystalline Langmuir monolayer may go through mesophases considered as a mixture of "clusters, ' i.e., domains of mesoscopic dimension. The size of the domains depends on the length of the molecular tails that determine the direct coupling for orientational ordering. The shorter the tails and the smaller the clusters, the easier they are fluidized, and the system approaches a 2D net. Thus, if any Langmuir monolayer structure would be hexaticlike, it is only probable for those formed by short tail amphiphiles. A schematic diagram suggested by Kats and Lajzerowicz clearly illustrates this point. ${ }^{13}$

In our approach, the hexagonal, high-pressure (LS) phase is treated as the quasi-2D solid of highest symmetry that serves as the parent phase for others. The coupling between the translational and elastic subsystem of head groups and the rotational one of tails has been described in terms of microscopic translational-rotational coupling parameters. ${ }^{3}$ The phases are characterized by both strain and orientational parameters, employing spherical harmonics that are related to each other through the elasticity of the system. This approach suggests that the orientational fluctuations of the molecular tails can be conveniently described by the concept of elastic dipoles embedded in an isotropic 2D elastic medium. ${ }^{9}$

Our recent studies have discussed the swiveling transition $^{14}$ in terms of a strain-tilt-backbone order parameter and have shown how the molecular cross-section or backbone orientation can be represented by an internal strain. ${ }^{11}$ The analysis of the resulting free energy surface spanned in the order parameter space indicated that the swiveling transition is between the $L_{2}$ phase (a rectangular lattice with molecules tilted towards nearest neighbors, NN) and the phase denoted as $L_{2}^{\prime}$ (a rectangular lattice characterized by molecular tilt ordering towards next nearest neighbors, NNN). Each of the phases may be viewed as an ordered phase, i.e., a defect-ordered structure due to orientational strain relaxation of a mixture of three types of domains characterized by strains, $\varepsilon_{2} ;-1 / 2 \varepsilon_{2}+\sqrt{3} / 2 \varepsilon_{6} ;-1 / 2 \varepsilon_{2}$ $+\sqrt{3} / 2 \varepsilon_{6}$, which are directly related to the tilt-backbone orientation of the molecules. The swiveling transition is then interpreted as a ferroelastic transition ${ }^{11}$ corresponding to a strain reversal, e.g., $\varepsilon_{2}=-\varepsilon_{2}$.

In this contribution, Langmuir monolayers are discussed from the perspective of elasticity theory and previous studies are expanded. ${ }^{9}$ Disorder of the molecular tails is treated as "defects" characterized as elastic dipoles grafted onto a 2D elastic medium. The orientational ordering appears at three levels: tilt, backbone orientation, and domain formation. The elastic interaction between the "defects" is considered and the nature of disorder in the parent LS phase is specifically analyzed. This approach has been strongly guided by a discussion by Sirota, ${ }^{4}$ who has suggested that the LS phase "has macroscopic hexagonal symmetry", with molecules packed in a distorted structure. Treating the LS phase as a mixture of rectangular and/or oblique domains, i.e., rectangular, oblique defects within a 2D elastic medium, we analyze density fluctuations due to elastic interactions between the domains. The correlation function is calculated for the elastic dipoles, which characterize the domains. The way the elastic interaction renormalizes the elastic properties of the mesophases is also discussed. Results are examined in the context of recent experiments on the microscopic ${ }^{7}$ and macroscopic ${ }^{8}$ elasticity of the monolayers and kinetic aspects of the elastic response are also considered. New studies of the positional disorder in LS, and $\mathrm{S}$ phases ${ }^{6}$ stimulate an additional discussion on the contribution of the elastic domains to the $\mathrm{x}$-ray diffuse scattering.

\section{THE MODEL}

A monolayer system is modeled as orientationally free tails grafted onto an elastic 2D net formed by the head groups of the amphiphilic molecules. Following the microscopic derivation developed previously, the system energy is described as $^{3}$

$$
V(\{\varepsilon\},\{\Omega\})=V^{R}(\{\Omega\})+V^{T R}(\{\varepsilon\},\{\Omega\})+V^{T}(\{\varepsilon\}) .
$$

The orientational part of the energy, $V^{R}(\{\Omega\})$, requires comment. Clearly, the LS phase, which is treated as disordered, should have an orientational probability distribution function for the molecular tails that is continuous and fairly uniform. In view of this, the orientational potential has been previously described in terms of continuous rotator functions, surface harmonics, adapted for the $C_{6 \mathrm{v}}$ symmetry of the LS phase. ${ }^{3}$ These are $Y_{1} \approx \eta \cos \phi$ and $Y_{2} \approx \eta \sin \phi$, which belong to the doubly degenerate $E_{1}$ representation, $Y_{3}$ $\approx\left(3 \cos ^{2} \phi-1\right)$ which belongs to the totally symmetric representation and $Y_{4} \approx \eta^{2} \cos 2 \phi$ and $Y_{5} \approx \eta^{2} \sin 2 \phi$ which transform as components of the doubly degenerate $E_{2}$ representation. This set of surface harmonics describes orientational fluctuations in terms of tilt $(\eta=\sin \theta)$ and azimuthal $(\phi)$ angles. Thermal averages of the harmonics $\langle Y\rangle$ serve as orientational order parameters.

The translational part of the energy, $V^{T}(\{\varepsilon\})$, is conveniently described in terms of the strain tensor components, $\left\{\varepsilon_{i j}\right\}$, for the 2D net. The variables, $e_{x x}-e_{y y}=\varepsilon_{2}$ and $e_{x y}$ $=\varepsilon_{6}$, transform as components of the $E_{2}$ representation, while $e_{x x}+e_{y y}=\varepsilon_{1}$ measures isotropic strain and transforms 
as the totally symmetric representation. The nonsymmetric strain variables for the $C_{6 \mathrm{v}}$ symmetry reference cell can be represented as

$$
\begin{aligned}
& \varepsilon_{2}=\xi \cos 2 \beta ; \quad \varepsilon_{6}=\xi \sin 2 \beta ; \\
& \xi=2 a_{1} / \sqrt{3} a_{0}\left[\sin \gamma_{1}-\sqrt{ } 3 \cos \gamma_{1}\right],
\end{aligned}
$$

where the distortion amplitude is expressed in terms of hexagonal lattice parameters, with $a_{0}$ the lattice constant before deformation. The angle $\beta$ defines the direction of the lattice deformation, and for $\beta=0, \pi, \ldots$, it is along the nearestneighbor (NN) direction, contracting for $\xi<0$ and stretching for $\xi>0$. The strain $\varepsilon_{2} \neq 0$ describes a deformation of the hexagonal net to a rectangular one, while $\varepsilon_{6} \neq 0$ defines distortion to an oblique lattice.

The important part of this model is the bilinear coupling between tensorial order parameters, the orientational fluctuations, and strains. The fluctuations described by parameters $\left\langle Y_{1}\right\rangle$ and $\left\langle Y_{2}\right\rangle$ are responsible for transitions to tilted phases within hexagonal nets, and because they do not couple linearly with strains, we neglect them. This is appropriate since we seek a disorder in the parent, hexagonal phase that is consistent with our model requiring that the disorder is due to elastic distortions, thus assuring strain plays an important role. The bilinear coupling of the type $\varepsilon_{2}\left\langle Y_{4}\right\rangle+\varepsilon_{6}\left\langle Y_{5}\right\rangle$ is considered in this treatment.

Taking $\left\langle Y_{4}\right\rangle$ and $\left\langle Y_{5}\right\rangle$ as the orientational order parameters, and $\varepsilon_{2}$ and $\varepsilon_{6}$ as related strains, the free energy function is

$$
F=F^{R}+F^{T R}+F^{T},
$$

where

$$
\begin{aligned}
F^{R}= & a(T)\left[\left\langle Y_{4}\right\rangle^{2}+\left\langle Y_{5}\right\rangle^{2}\right]+b\left[\left\langle Y_{4}\right\rangle^{3}-3\left\langle Y_{4}\right\rangle\left\langle Y_{5}\right\rangle^{2}\right] \\
& +c\left[\left\langle Y_{4}\right\rangle^{4}+\left\langle Y_{5}\right\rangle^{4}\right]+\cdots, \\
F^{T R}= & \alpha\left[\varepsilon_{2}\left\langle Y_{4}\right\rangle+\varepsilon_{6}\left\langle Y_{5}\right\rangle\right], \\
F^{T}= & 1 / 2 C_{66}^{0}\left[\varepsilon_{2}^{2}+\varepsilon_{6}^{2}\right]+A\left[\varepsilon_{2}^{3}-3 \varepsilon_{2} \varepsilon_{6}^{2}\right]+B\left[\varepsilon_{2}^{4}+\varepsilon_{6}^{4}\right]+\cdots .
\end{aligned}
$$

As the orientational and strain order parameters are equivalent, we choose the strain parameters as primary ones. Minimization of the free energy with respect to orientational fluctuations gives

$$
\begin{aligned}
F= & 1 / 2 C_{66}(T)\left[\varepsilon_{2}^{2}+\varepsilon_{6}^{2}\right]+A(T)\left[\varepsilon_{2}^{3}-3 \varepsilon_{2} \varepsilon_{6}^{2}\right] \\
& +B(T)\left[\varepsilon_{2}^{4}+\varepsilon_{6}^{4}\right]+\cdots,
\end{aligned}
$$

where

$$
C_{66}(T)=C_{66}^{0}-\alpha^{2} / a(T),
$$

is the renormalized elastic constant. This renormalization is due to orientational fluctuations at the molecular level, and it is the expected result of translation-rotational coupling. ${ }^{3}$ The temperature dependence, which enters via the $A(T)$ coefficient, may arise from either or both of the following contributions: (1) an effective orientational field experienced by a single molecular tail in the environment of its neighbors which is temperature dependent; and (2) an orientational entropy contribution due to a change in the orientational distri- bution function. In other words, it is an orientational disorder on the molecular scale that renormalizes the elasticity of the monolayer.

This disorder will be briefly discussed. An orientational disorder described by the tensorial order parameters, $\left\langle Y_{4}\right\rangle$ and $\left\langle Y_{5}\right\rangle$ is required. It can be due to fluctuations in tilt of cylindrical (rotating) molecules and/or fluctuations in molecular backbone orientation. For the tilt fluctuations, which can be visualized as precessionlike fluctuations, the order parameters are directly related to tilt angle via $\eta=\sin \theta, Y_{4}$ $\approx \eta^{2} \cos 2 \phi$ and $Y_{5} \approx \eta^{2} \sin 2 \phi$. Fluctuations in backbone orientation may also be characterized by the same surface harmonics, say, $Y_{4} \approx \delta \cos 2 \psi$ and $Y_{5} \approx \delta \sin 2 \psi$, but in this case $\delta$ is a measure of a distortion in the effective cross section of a molecule from a circular one mapped by rotation. In a real system, such separation of contributions to the orientational disorder is highly artificial, and for this reason it is more convenient to interpret the orientational order parameters, $\left\langle Y_{4}\right\rangle$ and $\left\langle Y_{5}\right\rangle$, as components of local stress represented by the elastic dipoles that are due to both contributions. Thus, the suitable functions are $Y_{4} \approx \Delta \cos 2 \psi$ and $Y_{5}$ $\approx \Delta \sin 2 \psi$, with $\Delta=\left(\eta^{2}+\delta\right)$ indicating the short axis of an ellipse along the $\psi=0$ direction.

Thermal averages of the harmonics are determined by an orientational distribution function, and in the case where the function is approximated by three "pocket states" located at $\psi=0,2 \pi / 3,4 \pi / 3$, they determine the probabilities, $p_{i}$, that molecular tails are within a given state

$$
\begin{aligned}
& p_{1}=1 / 3+2 / 3\left\langle Y_{4}\right\rangle, \\
& p_{2}=1 / 3-1 / 3\left\langle Y_{4}\right\rangle+1 / \sqrt{ } 3\left\langle Y_{5}\right\rangle, \\
& p_{3}=1 / 3-1 / 3\left\langle Y_{4}\right\rangle-1 / \sqrt{ } 3\left\langle Y_{5}\right\rangle .
\end{aligned}
$$

The probabilities for the three "pocket states" give three, equivalent, local, orientational structures. Under thermodynamic conditions appropriate for the mesophases of Langmuir monolayers, such structures are expected to be of mesoscopic sizes. Following the equivalence between orientational order parameters and the strains, the structures with local orientational order given by the probabilities, $p_{i}$, can be characterized by strains: $\varepsilon_{2},-1 / 2 \varepsilon_{2}+\sqrt{ } 3 / 2 \varepsilon$, $-1 / 2 \varepsilon_{2}-\sqrt{3} / 2 \varepsilon_{6}$. These elastic domains are determined by the free energy function, Eq. (7).

\section{ELASTIC DOMAINS AND THEIR INTERACTION}

The rotational mesophase, LS, with a global hexagonal structure with, on average, vertical molecules is assumed to be a mixture of mesoscopic local structures characterized by strains or elastic dipoles related to local structural stresses. This view is consistent with the determination that there is no long-range translational order in $2 \mathrm{D}$ systems, and that local equilibria compete with the global one, as indicated by kinetic processes in these systems as well as by findings that mesophases are more crystalline than hexatic. Moreover, there is growing evidence that microscopic properties of monolayers differ from their corresponding macroscopic ones as is clearly shown by their elasticity. ${ }^{8}$ Obviously, for 


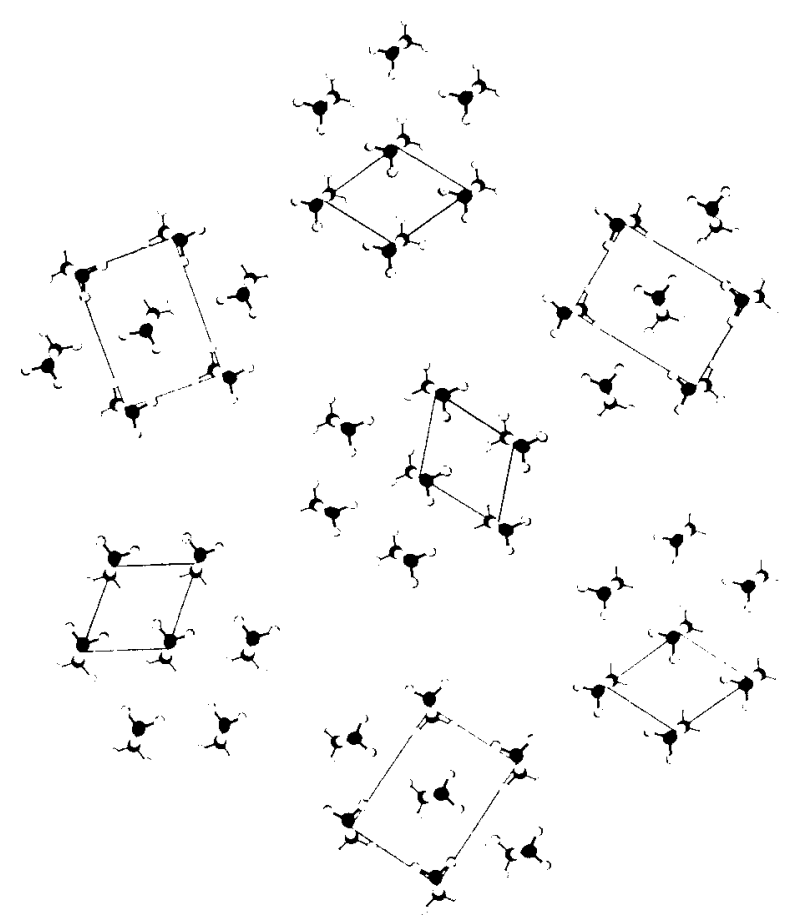

FIG. 1. Optimum local structures of rectangular and oblique domains grafted onto a global hexagonal net.

any renormalization of physical properties, one has to treat the mixture of domains as an interacting system.

Numerical calculations for structural optimization of Langmuir monolayers have already suggested that a mesophase can be considered as a mixture of domains. ${ }^{10,11}$ The potential energy landscape for a system with vertical molecules exhibits minima corresponding to elastic domains with small energy barriers for a reorientation. ${ }^{10}$ Moreover, the calculated energy minima for a two-molecule unit cell, thought to always contain amphiphiles in a herring-bone arrangement, is essentially identical to that found for a onemolecule unit cell, thereby making discrimination between these structures impossible. Results of the monolayer architecture minimization, in particular Figs. 7 and 8 of Ref. 10, can be taken as the optimum local structures for rectangular and/or oblique domains. When these structures are grafted onto a global, hexagonal net, as in Fig. 1, a schematic illustration of our model is obtained. It should be obvious from this, as well as our prior treatments, that this model is not restricted as to the nature of the amphiphile that it treats, e.g., only to diatomics.

We consider three types of domains that can be formed within a 2D hexagonal net. The domains are characterized by local stress tensors or, equivalently, elastic dipoles ${ }^{9}$ descriptive of the orientational fluctuations discussed above. The elastic dipole is represented as

$$
\mathbf{P}=P_{s}+P_{2} \mathbf{a}_{2}+P_{6} \mathbf{a}_{6},
$$

where

$$
\begin{aligned}
& P_{s}=1 / 2\left(P_{x x}+P_{y y}\right), \\
& P_{2}=1 / 2\left(P_{x x}-P_{y y}\right) \equiv\left\langle Y_{4}\right\rangle,
\end{aligned}
$$

$$
P_{6}=P_{x y} \equiv\left\langle Y_{5}\right\rangle
$$

and the unit matrices are

$$
\mathbf{a}_{2}=\begin{array}{cc}
1 & 0 \\
0 & -1
\end{array}, \quad \mathbf{a}_{6}=\begin{array}{ll}
0 & 1 \\
1 & 0
\end{array} .
$$

Since the domains are determined by symmetry elements lost by an ordering process, applying sixfold and threefold axis symmetry operations generates the local stress tensors for other domains. For example,

$$
\mathbf{P}^{(2)}=\mathbf{C}_{6} \mathbf{P}^{(1)} \mathbf{C}_{6}^{T} \quad \text { and } \mathbf{P}^{(3)}=\mathbf{C}_{3} \mathbf{P}^{(1)} \mathbf{C}_{3}^{T} \text {. }
$$

It should be emphasized that because the same symmetry is obtained for rectangular and oblique deformations, only three types of domains need to be considered. Each domain is, in principle, a combination of a totally symmetric strain associated with the change in a unit cell's line and rectangular and oblique deformations, respectively. Having specified the mesoscopic domains by the elastic dipoles, fluctuations in the density of the dipoles are now sought.

The density of a domain at point $\mathbf{x}$ in the system is designated as, $\rho_{0}(\mathbf{x})$. $\mathbf{x}$ thus locates a center of a local stress that is characteristic for a given domain. The mesophase, which is considered as a mixture of domains, is assumed to be in global thermodynamic equilibrium, i.e., in a stress-free state. ${ }^{15}$ At equilibrium, the density of domains of type $\alpha$ is

$$
\begin{aligned}
\rho_{\alpha}(\mathbf{x})= & \rho_{0}(\mathbf{x})\left[\exp \left(-\beta E_{\alpha}(\mathbf{x})\right) / V^{-1} \int d V \sum_{\alpha}\right. \\
& \left.\times \exp \left(-\beta E_{\alpha}(\mathbf{x})\right)\right],
\end{aligned}
$$

where $\beta=1 / k T$ ( $k$ is Boltzmann's constant), $V$ is the volume of the system, and $E_{\alpha}(\mathbf{x})$ is the energy of interaction of the domain in orientation $\alpha$ with the surrounding field formed by the distribution of domains. The energy is assumed to be purely elastic,

$$
E_{\alpha}(\mathbf{x})=-\mathbf{P}^{\alpha}(\mathbf{x}) \varepsilon(\mathbf{x})=-P_{i j}^{\alpha}(\mathbf{x}) \varepsilon_{i j}(\mathbf{x}),
$$

where $\varepsilon(\mathbf{x})$ is the effective strain acting at site $\mathbf{x}$. This is a sum of the macroscopic uniform strain, $\varepsilon$, and the local, mesoscopic strain, $\delta \varepsilon(\mathbf{x})$, due to surrounding mesoscopic elastic dipoles. Thus,

$$
\varepsilon_{i j}(\mathbf{x})=\varepsilon_{i j}+\delta \varepsilon_{i j}(\mathbf{x}),
$$

where

$$
\delta \varepsilon_{i j}(\mathbf{x})=\sum_{x \neq x^{\prime}} K_{i j l m}\left(\mathbf{x}, \mathbf{x}^{\prime}\right) p_{l m}\left(\mathbf{x}^{\prime}\right) .
$$

The elastic strain field, $K_{i j l m}\left(\mathbf{x}, \mathbf{x}^{\prime}\right)$, is just a correlation function for the mesoscopic strains in the uniform, disordered system,

$$
K_{i j l m}\left(\mathbf{x}, \mathbf{x}^{\prime}\right)=\beta\left\langle\delta \varepsilon_{i j}(\mathbf{x}) \delta \varepsilon_{l m}\left(\mathbf{x}^{\prime}\right)\right\rangle_{\text {unif }} .
$$

The density of elastic dipoles at site $\mathbf{x}^{\prime}$ is

$$
p_{l m}\left(\mathbf{x}^{\prime}\right)=\sum_{\alpha} \delta \rho_{\alpha}\left(\mathbf{x}^{\prime}\right) P_{l m}^{\alpha}\left(\mathbf{x}^{\prime}\right) .
$$

Now, the fluctuation in the elastic dipole density can be introduced,

$$
\delta \rho_{\alpha}(\mathbf{x})=\rho_{\alpha}(\mathbf{x})-\rho_{0}(\mathbf{x}),
$$


as the key parameter which determines local order. This parameter may be envisaged as equivalent to the orientational probabilities, $p_{i}$, expressed in Eq. (9). The difference is, however, that the elastic dipole density fluctuation measures an ordering of mesoscopic domains due to elastic interactions, while the orientational probabilities are determined for a homogeneous system within a domain. Disorder in the inhomogeneous system means that $\delta \rho_{\alpha}(\mathbf{x})=0$ at every site, while in a state with order, $\delta \rho_{\alpha}(\mathbf{x}) \neq 0$.

The elastic energy is now assumed to be smaller than the thermal energy. This requirement can be satisfied by taking the external strain to be sufficiently small [see Eq. (17)]. With this approximation and the global equilibrium condition, Eq. (16), the density fluctuation becomes

$$
\delta \rho_{\alpha}(\mathbf{x})=\beta\left[\rho_{0}(\mathbf{x}) / 3\right] P_{i j}^{\alpha}(\mathbf{x}) \varepsilon_{i j}(\mathbf{x}) .
$$

This relation is valid under the condition of a stress-free system and, in the case of Langmuir monolayers, the global equlibrium will appear after a system relaxes to such a state. This process is known to be rather slow, and will be taken as the reference state.

In the situation where an experiment on Langmuir monalyers is performed without allowing the system to relax to the global equlibrium, the condition for local equilibrium must be employed. This produces the following expression for the density fluctuation:

$\delta \rho_{\alpha}(\mathbf{x})=-\beta \rho_{0}(\mathbf{x}) / 3\left[P_{i j}^{\alpha}(\mathbf{x})-1 / 3 \sum_{\alpha} P_{i j}^{\alpha}(\mathbf{x})\right] \varepsilon_{i j}(\mathbf{x})$.

This equation demonstrates that, when a system is in local thermodynamic equilibrium within a domain, the density fluctuation of local stress is determined only by the deviatoric part of the elastic dipole. For further discussion, global equilibrium is assumed, although the same treatment can be executed for the case of local equilibrium.

\section{ELASTIC DIPOLE DENSITY CORRELATION}

The density fluctuation of the elastic dipoles shows [see Eq. (23)] a self-consistent dependence via the local strain field coupled to the elastic dipole density. The key quantity which couples the fluctuations is the elastic strain correlation function, $K_{i j l m}\left(\mathbf{x}, \mathbf{x}^{\prime}\right)$, which is a nonlocal compressibility tensor. Discussion of this function is presented in the Appendix, and, henceforth, the function is assumed known. Moreover, the limit of large distances between domains is assumed and the site indices are consequently dropped.

On introducing Eqs. (21), (19), and (18) into (23), the density fluctuation of elastic dipoles is given by

$$
\delta \rho_{\alpha}=\beta \rho_{0} / 3\left[\mathbf{1}-\beta \rho_{0} / 3 \mathbf{J}\right]_{\alpha \beta}^{-1} P_{i j}^{\beta} \varepsilon_{i j},
$$

where

$$
J_{\alpha \beta}=P_{i j}^{\alpha} K_{i j l m} P_{l m}^{\beta},
$$

is the elastic energy of interaction between domains of types $\alpha$ and $\beta$. The matrix is composed of values, $a=J_{\alpha \alpha}$ and $b$ $=J_{\alpha \beta}(\alpha \neq \beta)$. The correlation function for the elastic dipoles' density fluctuations can be deduced from Eq. (25). The susceptibility is

$$
\chi_{\alpha \beta}=\beta\left\langle\delta \rho_{\alpha} \delta \rho_{\beta}\right\rangle=\beta \rho_{0} / 3\left[\mathbf{1}-\beta \rho_{0} / 3 \mathbf{J}\right]_{\alpha \beta}^{-1} .
$$

The symmetry of the susceptibility matrix implies that there will be three eigenvalues:

$$
\chi_{A}=\left(\rho_{0} / 3\right)\left[\beta^{-1}-\beta_{c}^{-1}\left(A_{g}\right)\right]^{-1},
$$

for the totally symmetric representation, and

$$
\chi_{E}=\left(\rho_{0} / 3\right)\left[\beta^{-1}-\beta_{c}^{-1}\left(E_{2}\right)\right]^{-1},
$$

for the $E_{2}$ doubly degenerate representation. These eigenvalues can be conveniently expressed as critical temperatures:

$$
\begin{aligned}
& \beta_{c}^{-1}\left(A_{g}\right)=\left(\rho_{0} / 3\right)(a+2 b), \\
& \beta_{c}^{-1}\left(E_{2}\right)=\left(\rho_{0} / 3\right)(a-b),
\end{aligned}
$$

which determine the critical points for ordering of the elastic dipoles of the corresponding symmetries. At the temperatures given by Eqs. (30) and (31), compressibility and shear compliance of the systems become infinite, respectively. The latter instability is related to ferroelastic ordering.

For further discussion, the totally symmetric part of the elastic dipole is assumed to be zero. Taking into account the symmetries of the elastic dipoles for the three domains, the elastic interaction energies are calculated (see the Appendix) as

$$
\begin{aligned}
& a=\left(2 \pi \mu_{0}\right)^{-1} R^{-2}\left(P_{2}^{2}+P_{6}^{2}\right) ; \\
& b=-\left(4 \pi \mu_{0}\right)^{-1} R^{-2}\left(P_{2}^{2}+P_{6}^{2}\right),
\end{aligned}
$$

where $\mu_{0}$ is the shear elastic constant of the system in the disordered state. This result is consistent with the intuition that elastic domains in the same orientation repeal each other and prefer orthogonal orientations in their neighborhood. Notice, that due to specific features of the elastic interactions in the $2 \mathrm{D}$ net, the critical temperature for ordering of elastic domains, Eq. (31), is distance dependent. Ordering, therefore, appears as a continuous process with decreasing temperature with concomitant increase of the uniform domains' size. In terms of the $E_{2}$ eigenvalue, the density fluctuation of the elastic dipole type $\alpha$ is

$$
\delta \rho_{\alpha}=\chi_{E}(R) P_{i j}^{\alpha} \varepsilon_{i j},
$$

which is also distance dependent in the sense discussed above. Thus, the susceptibility shows the desired algebraic decay with the distance but without the constraint of hexatic structure.

\section{DISCUSSION}

There are notable consequences for the properties of monolayers that follow from this model that treats a mesophase as a mixture of elastic domains. A result of this is disorder associated with the domain formation. Thus it is necessary to examine the ramifications of the domain formation for a monolayer's elastic properties as well as for diffuse scattering of x-rays from it.

\section{Implications for elastic properties}

The elastic interaction between the domains causes an ordering process in the system, and this can create a change in macroscopic elastic properties. The case of a static response to an applied stress will be treated first. 
The formula for the elastic dipole density fluctuation, Eq. (25), may be rewritten as

$$
\delta \rho_{\alpha}=\beta \rho_{0} / 3 \chi_{\alpha \beta}(R) P_{i j}^{\beta} S_{i j l m}^{0} \sigma_{l m} .
$$

The macroscopic strain has been expressed in terms of the macroscopic stress tensor, $\sigma$, and the elastic compliance tensor for an isotropic medium,

$$
\begin{aligned}
S_{i j l m}^{0}= & \left(1 / 2 \mu_{0}\right)\left[1 / 2\left(\delta_{i l} \delta_{j m}+\delta_{i m} \delta_{j l}\right)\right. \\
& \left.-\left(\lambda_{0} /\left(3 \lambda_{0}+2 \mu_{0}\right)\right) \delta_{i j} \delta_{l m}\right] .
\end{aligned}
$$

The total strain experienced by the macroscopic system is the sum of the elastic $\left(\mathbf{S}^{0} \sigma\right)$ and plastic strains, i.e., the density of elastic dipoles. Written in tensor notation, this is

$$
\varepsilon=\mathbf{S}^{0}[\sigma+\mathbf{p}],
$$

where the elastic dipole density is

$$
\mathbf{p}=\mathbf{P}^{\alpha} \chi_{\alpha \beta}(R) \mathbf{P}^{\beta} \mathbf{S}^{0} \sigma .
$$

The total strain is then

$$
\varepsilon=\mathbf{S}^{0}\left[\mathbf{1}+\mathbf{P}^{\alpha} \chi_{\alpha \beta}(R) \mathbf{P}^{\beta} \mathbf{S}^{0}\right] \sigma,
$$

and one can identify the renormalized compliance

$$
\mathbf{S}=\mathbf{S}^{0}+\left(\mathbf{S}^{0} \mathbf{P}^{\alpha}\right) \chi_{\alpha \beta}(R)\left(\mathbf{P}^{\beta} \mathbf{S}^{0}\right) .
$$

The second term in Eq. (39) can be identified as the correlation function for strain fluctuations at large distances in the system of interacting elastic domains,

$$
\beta\langle\delta \varepsilon(R) \delta \varepsilon(0)\rangle=\left(\mathbf{S}^{0} \mathbf{P}^{\alpha}\right) \chi_{\alpha \beta}(R)\left(\mathbf{P}^{\beta} \mathbf{S}^{0}\right) .
$$

For the symmetry of the domains in our system, the shear elastic constant is renormalized according to the relation

$$
[\mu(R)]^{-1}=\mu_{0}^{-1}\left\{1+\mu_{0}^{-1}\left(P_{2}^{2}+P_{6}^{2}\right) \chi_{E}(R)\right\} .
$$

The $R$ dependence of the susceptibility is explicitly a function of the distance

$$
\chi_{E}(R)=\left(\rho_{0} / 3\right)\left[\beta^{-1}-\left(4 \pi \mu_{0}\right)^{-1} R^{-2}\left(P_{2}^{2}+P_{6}^{2}\right)\right],
$$

and correspondingly, the $R$-dependent shear elastic constant might be considered as being the macroscopic counterpart of the microscopic elastic constant. The renormalization of the shear elasticity is due to orientational relaxation of the elastic domains and such a relaxation will, obviously, influence the elastic response of a macroscopic system. One may expect that the difference between macroscopic and microscopic elasticity increases with lowering the temperature because of the temperature-induced ordering process. In a real system, the temperature interval studied is usually quite small and this elasticity difference may be difficult to observe.

An important aspect of the elasticity of Langmuir monolayers is that the elastic response can be, and often is, a nonequlibrium one. This means that the system responds to an applied stress within the relaxation time of the elastic domains. Thus, inquiry into the time evolution of the elastic domains and how the relaxation process influences the elastic response is necessary. The elastic dipole density fluctuation, which obtains at thermodynamic equilibrium, is given by Eq. (33) for the rectangular and/or oblique domains. This is denoted as the value at infinite time

$$
\delta \rho_{\alpha}(\infty)=\chi_{E} P_{i j}^{\alpha} \varepsilon_{i j} .
$$

Time evolution of the density fluctuation follows from the following kinetic equation:

$$
d\left[\delta \rho_{\alpha}(t)\right] / d t=-(1 / \tau)\left[\delta \rho_{\alpha}(t)-\delta \rho_{\alpha}(\infty)\right],
$$

where $\tau$ is a relaxation time for a process of ordering of the elastic domains. It might also be considered as the residence time for a system in an orientational potential well. The time evolution of the elastic dipole density fluctuation is

$$
\delta \rho_{\alpha}(t)=\delta \rho_{\alpha}(\infty)[1-\exp (-t / \tau)] .
$$

It is, in fact, an approximation that one relaxation time is used for the elastic domains' reorientation. Since the domains are, in general, rectangular, i.e., a $P_{2}$ elastic dipole, and/or oblique, a $P_{6}$ elastic dipole, two relaxation times may be introduced: one for rectangular and one for oblique distortions.

The elastic dipole density that describes the local stress will show this same time evolution and, following the derivation for the static case, the following time dependence of the shear elastic constant obtains

$$
\begin{aligned}
{[\mu(R, t)]^{-1}=} & \mu_{0}^{-1}\left\{1+\mu_{0}^{-1}\left(P_{2}^{2}+P_{6}^{2}\right) \chi_{E}(R)\right. \\
& \times[1-\exp (-t / \tau)]\} .
\end{aligned}
$$

It is evident that any instantaneous measurements (at $t=0$ ) will not detect an elastic response of the elastic dipole density fluctuations, since it is insensitive to the ordering process of the elastic domains. On the other hand, experiments on a time scale for a system to reach thermodynamic equilibrium will measure the shear elastic constant of a system where an ordering process takes place. Equation (45) may be rewritten so the macroscopic elastic constant can be expressed in terms of its microscopic counterparts

$$
\left[\mu_{\mathrm{MACRO}}(R)\right]^{-1}=\mu_{0}^{-1}\left\{1+\mu_{0}^{-1}\left(P_{2}^{2}+P_{6}^{2}\right) \chi_{E}(R)\right\} .
$$

It has been found that the ratio $\left(\mu_{\mathrm{MACRO}} / \mu_{0}\right)$ is of the order of $10^{-2} .{ }^{8}$ Such a large difference indicates that the renormalization of the elasticity due to an ordering process of elastic domains in mesophases is very important. The renormalized shear elastic constant may be represented by an approximate formula, derived from Eq. (45),

$$
\mu(R, t)=\mu_{0}-\left(P_{2}^{2}+P_{6}^{2}\right) \chi_{E}(R)[1-\exp (-t / \tau)] .
$$

It has been found ${ }^{16}$ that such a formula well describes the time-dependent shear elastic response of monolayers and that the ratio $\mu(R, t \rightarrow \infty) / \mu_{0}$ is of the order of $10^{-2}$. Within the model of elastic domains, it is not surprising that the ratio coincides with that for the macroscopic and microscopic elastic constants, as both are determined by the same renormalization factor.

For dynamical experiments performed within specific frequency regimes, the frequency dependent susceptibility can be introduced,

$$
\chi_{E}(R, \omega)=\chi_{E}(R)(1+i \omega \tau)^{-1},
$$

which reflects the relaxation process of ordering domains. Using this susceptibility, the renormalized elastic constant becomes frequency dependent, 


$$
[\mu(R, \omega)]^{-1}=\mu_{0}^{-1}\left\{1+\mu_{0}^{-1}\left(P_{2}^{2}+P_{6}^{2}\right) \chi_{E}(R, \omega)\right\},
$$

and, following standard notation, is written as

$$
\mu(\omega)=\mu^{\prime}(\omega)+i \omega \eta(\omega) .
$$

The real part of the shear elastic constant is

$$
\mu^{\prime}(\omega)=\mu_{0}\left\{1+\mu_{0}^{-1}\left(P_{2}^{2}+P_{6}^{2}\right) \chi_{E}(R)\left(1+\omega^{2} \tau^{2}\right)^{-1}\right\}^{-1},
$$

where we note that the shear elastic constant $\mu_{0}$ can be taken as a high-frequency property.

The imaginary part of the shear elasticity is expressed in terms of shear viscosity,

$$
\eta(\omega)=\left[\mu^{\prime}(\omega)\right]^{2} \mu_{0}^{-2}\left(P_{2}^{2}+P_{6}^{2}\right) \chi_{E}(R)\left(1+\omega^{2} \tau^{2}\right)^{-1} .
$$

This expression shows that the viscoelasticity of the system changes in the same way as does the real part of the shear elastic constant. Moreover, in the regime of Langmuir monolayer mesophases, where elastic domains possess orientational freedom, the viscoelasticity and the shear elastic constant show minimum values. Our findings explain the observations of "viscosity" made by Copeland et al., ${ }^{17}$ and by other, more recent, experiments on shear elasticity of Langmuir monolayers. $7,8,16$

\section{Implications for diffuse scattering}

Clearly, the "anomalous" elastic properties of the mesophases are related to disorder due to the existence of mesoscopic elastic domains. This disorder is seen in x-ray scattering experiments and manifests itself in the anomalous broadening of diffraction peaks at temperatures around the $L S / S$ transition. ${ }^{6}$ This observation has been intuitively connected with elastic properties, ${ }^{6,18}$ and shown to be in close analogy with bulk alkanes. ${ }^{4}$ However, even in a recent analysis of the positional disorder, ${ }^{6}$ it has not been shown how to quantitatively relate the x-ray scattering observations to the elastic properties. Our results, here and elsewhere, suggest the model of elastic domains is the only current one that can provide the basis for a consistent and coherently extendable physical picture of Langmuir monolayers. To further demonstrate the efficacy of this model, it will be extended to analyze $x$-ray diffuse scattering results and to calculate the contribution due to positional disorder induced by the elastic domains.

For the purpose of the diffuse scattering, the Fourier transform of the density fluctuation, $\delta \rho_{\alpha}(\mathbf{q})$ is used. In fact, the wave vector $(\mathbf{q})$-dependent density fluctuation can be considered as an analogue of the order parameter in the Landau theory of "weak crystallization.", 13,19 According to that theory, crystallization appears when a system becomes unstable against formation of a density fluctuation wave with a given wave vector. In a system of disordered elastic domains, an ordering process corresponds to "crystallization." It appears as a formation of a static distribution of elastic dipoles according to a pattern of density fluctuations corresponding to the lowest eigenvalue of the q-dependent susceptibility

$$
\chi_{\alpha \beta}(\mathbf{q})=\beta\left\langle\delta \rho_{\alpha}(\mathbf{q}) \delta \rho_{\beta}(\mathbf{q})\right\rangle .
$$

Conceptually, our approach using elastic dipole density fluctuations is similar and consistent with "weak crystallization" theory. In our model, the "crystallization" of elastic domains may be considered as a kind of spinodal decomposition process.

According to the kinematic theory, the x-ray scattering intensity at point $\mathbf{Q}=\mathbf{q}+2 \pi \mathbf{H}$, where $\mathbf{H}$ is a reciprocal lattice vector, is expressed by

$$
I_{\text {dif }}(\mathbf{Q})=\left\langle|\Delta \varphi(\mathbf{q})|^{2}\right\rangle
$$

where $\Delta \varphi(\mathbf{q})$ is a fluctuation in the scattering amplitude at point $\mathbf{q}$ of the reciprocal lattice. For a system composed of rectangular and/or oblique domains, the fluctuation is due to the elastic dipole density fluctuation $\delta \rho_{\alpha}(\mathbf{q})$. The fluctuation causes inhomogeneous displacements, $\mathbf{u}(\mathbf{q})$, which may be expressed as a response to a fluctuating force, $\mathbf{V}^{\alpha}(\mathbf{q})$, produced by a given elastic domain

$$
\mathbf{u}(\mathbf{q})=\mathbf{G}(\mathbf{q}) \sum_{\alpha} \mathbf{V}^{\alpha}(\mathbf{q}) \delta \rho_{\alpha}(\mathbf{q}) .
$$

$\mathbf{G}(\mathbf{q})$ is the elastic Green's function specified in the Appendix. Assuming the displacements are small, the contribution to the $\mathrm{x}$-ray diffuse scattering is

$$
I_{\text {dif }}(\mathbf{Q})=\beta^{-1} F_{\alpha}(\mathbf{q}) \chi_{\alpha \beta}(\mathbf{q}) F_{\beta}(-\mathbf{q}),
$$

where

$$
F_{\alpha}(\mathbf{q})=\left[\Delta f_{\alpha}+f \mathbf{Q G}(\mathbf{q}) \mathbf{V}^{\alpha}(\mathbf{q})\right] .
$$

$\Delta f_{\alpha}$ is the difference between scattering factors for different types of the elastic domains and the reference, disordered system. Simplifications which may be imposed on the formula are the following: (1) approximation of the susceptibility by its value calculated for "large $R$ '" and use of Eq. (27); (2) expression of the force, within the same limit, in terms of the elastic dipoles. Then,

$$
\mathbf{V}^{\alpha}(\mathbf{q}) \propto i \mathbf{v} \mathbf{P}^{\alpha} \mathbf{q} \text {. }
$$

With these approximations, the contribution to x-ray diffuse scattering due to positional disorder caused by the rectangular and/or oblique elastic domains is

$$
\begin{aligned}
I_{\mathrm{dif}}(\mathbf{Q})= & \beta^{-1} \chi_{\alpha \beta}\left[\Delta f_{\alpha}+f \mathbf{Q G}(\mathbf{q}) \mathbf{P}^{\alpha} \mathbf{q}\right] \\
& \times\left[\Delta f_{\beta}+f \mathbf{Q G}(\mathbf{q}) \mathbf{P}^{\beta} \mathbf{q}\right] .
\end{aligned}
$$

This formula may be further rearranged, after some approximations, to show that

$$
I_{\text {dif }}(\mathbf{Q}) \propto \beta^{-1}\left(\mathbf{S}^{0} \mathbf{P}^{\alpha}\right) \chi_{\alpha \beta}\left(\mathbf{P}^{\beta} \mathbf{S}^{0}\right) .
$$

This shows that the contribution to the diffuse scattering is proportional to that which renormalizes the elasticity of the system. Thus, observations of anomalous elasticity and anomalous scattering are closely related by the same mechanism of elastic domain ordering. It is evident that the scattering is proportional to the square of the elastic dipole, i.e., the local stress, which, in a product with the density fluctuation, serves as an order parameter. This has intuitively been assumed in the recent analysis of positional disorder in LS/S phases. ${ }^{6}$ This example shows how elastic domains can be employed to understand $\mathrm{x}$-ray scattering experiments for bulk alkanes, and, indeed, such a model-based analysis has been sought. ${ }^{4}$ 


\section{CONCLUSIONS}

Consideration of recent discussions on the ordering of Langmuir monolayer mesophases ${ }^{4,6}$ has led us to develop a consistent phenomenological model based on the hexagonal, parent phase, LS, of alkanoic acid Langmuir monolayers. Within this picture, the phase is composed of clusters or domains that have local equilibrium structures, rectangular and/or oblique. Orientational ordering is assumed to be at two levels, molecular and domain. At the molecular level, local structures are formed that are determined by the interplay of molecular translational-rotational coupling and direct van der Waals interaction between tails. ${ }^{3}$ Orientational order of the clusters arise from elastic interaction between the domains, characteristic for lower symmetry than for the global symmetry of the LS phase. We have chosen the simplest situation of three rectangular and/or oblique domains and characterized them by local stresses-the elastic dipoles. Correlation between the domains due to the elastic interaction has been considered.

Several results of the model have been obtained that relate directly to current observations, some of which, until now, have resisted explanation. The elastic shear response of a macroscopic system is expected to be smaller than the microscopic one due to the elastic orientational relaxation of the domains. This explains an effect already observed. ${ }^{7,8}$ Moreover, we have considered the time-evolution and frequency dependence of the elastic response and offered interpretation of the difference between the observed static and high-frequency shear elasticity, ${ }^{16}$ as well as for anomalous shear viscosity. ${ }^{17}$

Positional order of the monolayers, as observed by $\mathrm{x}$-ray scattering, ${ }^{6}$ may be expected to be influenced by the disorder of the clusters and their correlation. We have shown how the elastic domains cause positional disorder and contribute to $\mathrm{x}$-ray diffuse scattering. This scattering intensity is shown to be proportional to the local strain correlation function and depends on the product of elastic dipoles that are the measures of local stresses. These considerations provide a model-based explanation for the observed anomalous broadening of the diffraction peaks that have been recently reported. ${ }^{6}$

Systems where small enough clusters are formed and monolayers are either close to a solid-liquid transition or comprised of amphiphiles with rather short molecular tails associated with vanishingly small elastic dipoles. Therefore, the positional disorder will be at a molecular level and one would expect an exponential decay as predicted for a purely 2D net. However, for molecules with longer tails, and thereby able to form larger clusters and mesoscopic domains, the scattering experiment detects their correlation function which decays algebraically and which is symptomatic of the crystalline state for the monolayers.

The phenomenological picture we suggest serves as a bridge between more rigorous theories for 2D systems. These have been described and compared by Kats and Lejzerowicz ${ }^{13}$ who conclude that the Landau "weak crystallization" concept is more suitable for amphiphiles with long tails which are expected to have a clear solid-liquid transition. On the other hand, the theory of melting by the dislo- cation dissociation mechanism, which predicts the hexatic phase, is more suitable for systems with shorter tail amphiphiles. Within our picture this might be mapped onto a scale of clusters that are dependent on the direct tail-tail interaction. This brief treatment indicates how the "weak crystallization" concept can be understood within our phenomenological model.

In general terms, the model we offer treats Langmuir monolayers as frustrated systems, where a local structure and its associated equilibria competes with a global structure and its associated equilibria. Speaking in terms of competing interactions, the effective rotational coupling between tails (van der Waals and translational-rotational coupling at a molecular level) which form clusters of lower symmetry, competes with long-range elastic interactions between the elastic domains. The results of this study further support the generality, efficacy, and usefulness of our model that treats mesophases of Langmuir monolayers as disordered solids. In this, as well as earlier treatments, $3,10,11$ we have endeavored to show how the elastic solid theory of Langmuir monolayers is a natural, easily extended approach that offers a completely consistent and unifying structure for understanding a wide variety of experimental findings for Langmuir monolayers.

\section{ACKNOWLEDGMENTS}

T.L. thanks the Region de Nord Pas de Calais for supporting part of the research and his stay at L.D.S.M., Universite de Lille 1. Partial support has also been provided by the Polish Committee for Scientific Research for cooperation with France (POLONIUM), and by the U.S. Army Research Office (DEPSCOR Grant No. DAAH 04-96-1-0394).

\section{APPENDIX}

Here we discuss the correlation function that couples local stresses, the elastic dipoles. The function may be written in terms of wave vector, $\mathbf{q}$, transformation as

$$
K_{i j l m}\left(\mathbf{x}, \mathbf{x}^{\prime}\right)=1 / N \sum_{q} G_{i j l m}(\mathbf{q})\left[\exp i \mathbf{q}\left(\mathbf{x}-\mathbf{x}^{\prime}\right)-1\right],
$$

where

$$
G_{i j l m}(\mathbf{q})=\beta\left\langle\delta \varepsilon_{i j}(\mathbf{q}) \delta \varepsilon_{l m}(-\mathbf{q})\right\rangle_{\text {unif }}
$$

is the correlation function of strain fluctuations in a uniform (disordered) system. The second term in square brackets assures that the self-term, $K_{i j l m}\left(\mathbf{x}_{=} \mathbf{x}^{\prime}\right)$, is excluded from the elastic coupling. This term gives rise to the energy of creation of an elastic dipole in an elastic medium. The strain fluctuation correlation function is

$$
G_{i j l m}(\mathbf{q})=q_{i} q_{l} G_{j m}(\mathbf{q}),
$$

where $G_{j m}(\mathbf{q})$ is the displacement-displacement correlation function. For an isotropic elastic medium, an appropriate approximation for a hexagonal net, and in the limit of small $\mathbf{q}$,

$$
\begin{aligned}
G_{j m}(\mathbf{q}) & =\beta\left\langle u_{j}(\mathbf{q}) u_{m}(-\mathbf{q})\right\rangle \\
& =\left(1 / q^{2} \mu_{0}\right)\left[\delta_{j m}-\left(\lambda_{0}+\mu_{0}\right) /\left(\lambda_{0}+2 \mu_{0}\right) n_{j} n_{m}\right],
\end{aligned}
$$


where $\lambda_{0}$ and $\mu_{0}$ are Lamé's constants and $n_{I}=q_{i} / q$.

Alternatively, the strain field can be calculated as

$$
K_{i j l m}\left(\mathbf{x}, \mathbf{x}^{\prime}\right)=\left(\partial / \partial R_{i}\right)\left(\partial / \partial R_{l}\right) G_{j m}(\mathbf{R}),
$$

where $\mathbf{R}=\mathbf{x}-\mathbf{x}^{\prime}$ and

$$
G_{j m}(\mathbf{R})=1 / N \sum_{q} G_{j m}(\mathbf{q})\left[\exp i \mathbf{q}\left(\mathbf{x}-\mathbf{x}^{\prime}\right)-1\right]
$$

Summation over $\mathbf{q}$ space is done by integration over $2 \mathrm{D}$ space and for the calculations it is important to specify the elastic Green's function, the inverse of dynamical matrix. At this point, it must be decided how to treat the translational subsystem formed by the head groups. If treated as a purely $2 \mathrm{D}$, elastically isotropic net without interaction with the water substrate, then (A4) is introduced into (A6). This yields the well-known displacement-displacement correlation function for a $2 \mathrm{D}$ net,

$$
\left\langle u_{j}(0) u_{l}(R)\right\rangle_{2 \mathrm{D}} \approx-(B / 4 \pi \beta) \delta_{j l} \ln (R / a),
$$

in the limit of large $R$ ( $a$ is the order of the lattice spacing). Expressing the constant $B$ in terms of the elastic constants,

$$
B=1 / \mu_{0}+1 /\left(2 \mu_{0}+\lambda_{0}\right) \text {. }
$$

For our simple model, we also assume the limit of large distances. This can be justified within the picture that a monolayer domain represents a cluster of tails which are tilted towards (or opposite to) a domain center. This arises from geometric frustration due to the incompatibility of tail and head cross-sectional areas, as mentioned in the Introduction. The interactions of the domains is assumed to be elastic, therefore the coupling is between centers of the domains' stress, i.e., between the geometrical centers of domains. Thus, for reasonably sized domains, the limit of large distances between the stress centers can be justified. In this limit, the correlation function $K_{i j l m}\left(\mathbf{x}, \mathbf{x}^{\prime}\right)$, is denoted as $K_{i j l m}$ and, for the symmetries of the elastic dipoles considered, will be approximated by

$$
\beta\left\langle\delta \varepsilon_{i j}(\mathbf{x}) \delta \varepsilon_{l m}\left(\mathbf{x}^{\prime}\right)\right\rangle_{\mathrm{unif}}=K_{i j l m} \approx\left(4 \pi \mu_{0}\right)^{-1} R^{-2} \delta_{j m} \delta_{i l} .
$$

This result, although approximate, shows that correlation between local strain fluctuations within a uniform 2D net decay algebraically, as do orientational fluctuations. It is important to notice that the tensor, $\mathbf{K}$, although considered in the limit of large distances, does not correspond to the compliance tensor, $\mathbf{S}^{0}=\mathbf{C}^{-1}$, the inverse of the elastic constant tensor, as it does for a three-dimensional system.

${ }^{1}$ C. M. Knobler and R. C. Desai, Annu. Rev. Phys. Chem. 43, 207 (1992).

${ }^{2}$ A. M. Bibo, C. M. Knobler, and I. R. Peterson, J. Phys. Chem. 95, 5591 (1991).

${ }^{3}$ T. Luty and C. J. Eckhardt, J. Phys. Chem. 99, 8872 (1995).

${ }^{4}$ E. B. Sirota, Langmuir 13, 3847 (1997).

${ }^{5}$ D. Jacquemin et al., Angew. Chem. Int. Ed. Engl. 31, 130 (1992).

${ }^{6}$ V. M. Kaganer et al., Phys. Rev. Lett. 81, 5864 (1998).

${ }^{7}$ C. Zakri et al., Phys. Rev. B 55, 14163 (1997).

${ }^{8}$ C. Zakri, A. Renault, and B. Berge, Physica B 248, 208 (1998).

${ }^{9}$ T. Luty and C. J. Eckhardt, J. Phys. Chem. 100, 6793 (1996).

${ }^{10}$ D. R. Swanson, T. Luty, and C. J. Eckhardt, J. Chem. Phys. 107, 4744 (1997).

${ }^{11}$ T. Luty, D. R. Swanson, and C. J. Eckhardt, J. Chem. Phys. 110, 2606 (1999).

${ }^{12}$ I. R. Peterson and R. M. Kenn, Langmuir 10, 4645 (1994).

${ }^{13}$ E. I. Kats and J. Lajzerowicz, J. Exp. Theor. Phys. 83, 495 (1996).

${ }^{14}$ M. K. Durbin et al., J. Chem. Phys. 106, 8216 (1997).

${ }^{15}$ R. Siems, Phys. Status Solidi 42, 105 (1970).

${ }^{16}$ B. M. Abraham, K. Miyano, J. B. Ketterson, and S. Q. Xu, Phys. Rev. Lett. 51, 1975 (1983).

${ }^{17}$ L. E. Copeland, W. D. Harkins, and G. E. Boyd, J. Chem. Phys. 10, 357 (1942).

${ }^{18}$ M. C. Shih et al., Phys. Rev. A 45, 5734 (1992).

${ }^{19}$ V. M. Kaganer and E. B. Loginov, Phys. Rev. Lett. 71, 2599 (1993). 